\title{
32 AN OBJECT BASED VIRTUAL REALITY SIMULATION TOOL FOR DESIGN VALIDATION OF A NEW PARADIGM CLOTH MANUFACTURING FACILITY
}

\author{
Fabio Bonsignorio \\ PMARlab DIMEC University of Genova \\ bonsignorio@dimec.unige.it \\ Rezia Molfino \\ PMARlab DIMEC University of Genova \\ molfino@dimec.unige.it
}

\begin{abstract}
Within Leapfrog project, the general task is to design an effective and efficient manufacturing solution coping with the needs of the mass customization competitive scenario, overcoming the limitations which have so far prevented the automation of the garment assembly, through a comprehensive and general ('holistic') redesign of the cloth manufacturing process properly exploiting flexible automation and robotic technologies. Virtual Reality simulation is a core part of the interactive validation of design choices. The garment assembly line simulator has various objectives: (a) Validation of the garment assembly line 'holistic concept'; (b) Layout definition against realistic production data; (c) Prediction of realistic production metrics after line deployment. The simulator has been developed paying a special attention to easiness of maintenance: adding new resources or reports or changing the statistic of the production representative measures must require a limited effort; VR representation of what-if scenarios make easier comprehensive evaluation of aliernative layout solutions.
\end{abstract}

\section{INTRODUCTION}

The Leapfrog (Leadership for European Apparel Production From Research along Original Guidelines) project is a major European project aiming at a complete textile/clothes supply chain and manufacturing process redesign in order to reestablish European leadership in the textile industry.

Although the textile industry represents perhaps the oldest example of modern industrialization (the first cotton mill at Cromford, Derbyshire, UK, is usually considered the first example of a modern factory) the level of automation reached in this field of manufacturing engineering is far to be completed.

The process leading from raw materials to fabric rolls is already highly automated, on the contrary the final assembly of garments after the cutting of the fabric to the final sewing and finishing of the garment are currently performed by human workers: this is one of the main causes of the ongoing outsourcing process in the textile industry. 
A holistic radically new textile/clothes production paradigm is needed.

The system to be designed is a complex non linear manufacturing system: the relation between the design parameters and the constraint cannot be expressed in a closed form.

This is a typical case where the simulation of the proposed alternative design solutions is the main quantitative support for the design of the production facility $[1,3,6,7,8]$.

As what it is needed is a disruptively new layout of a complex mechatronic manufacturing system.

The new system is based on strong innovation of state-of-the-art solutions and new solutions:

1. Fast and automated single-ply cutting through multiple cutting heads: multiple plies cutting needs human, manually performed, separation of cut parts.

Instead of a single cutting head and multiple fabric plies we have single ply and multiple cutting heads, but we eliminate the need for human intervention

2. Fusing of all cut parts

3. Automatic robotic pick up of cut parts on the single ply cut table (see point 1) and transfer to an automatic hanging transport system

4. Automated 3D manipulation of the parts to prepare for the 3D sewing

5. Sewing process with:

- new 3-D-robot-sewing-technology and

- traditional sewing technique (different automation levels)

This is a key issue as sewing of the garment is currently done operating manually 2D sewing machines quite similar to the original century old Singer patent.

This system is thought as a first step toward achieving a high degree of mass customization. This relies on deploying a system that is flexible enough to lead to varied batches of mass production.

This is possible through the introduction of robotic technologies in several steps of the, reshaped, production process: these technologies allow the automation of operations that were not so far possible to automate, and, on the other hand, being the robots programmable machines, it is possible to have the benefits of mass production in a very versatile easily reprogrammable environment, enabling the so called 'mass customization'.

The system is devised to overcome most of the drawbacks of the current state of the art production methods by the creation of more advanced and rather breakthrough components or by devising methodologies that totally bypass those restrictions.

A simulation tool, named GALsim (Garment Assembly Line simulator), has been developed in order to support the design and assess the technologies to be used $[2,4,10,11]$.

The development of a new dedicated simulation tool was needed since the resource involved and the problems addressed are quite new, as GALsim has the objective of modeling a new paradigm cloth manufacturing facility.

It is an innovative simulation tool for an innovative production system design.

This simulation tool, based on Delmia Quest software, is a modular and scalable event-driven object based simulator and allows realistic VR representation of whatif scenarios. 


\subsection{Intended use of the simulator}

The simulation technology plays a key role in the solution design and in the technology assessment allowing the quantitative, and also 'visually animated' in VR, verification of the design choices and allowing to test in advance if the different proposed technologies can fit in the overall framework.

As the overall garment assembly process is being radically redesigned, there are many design variables, with a wide spectrum of new resource kinds, based on new robotics and mechatronic technologies and on new physical-chemical processes.

The new garment assembly line design is greatly helped, and perhaps made possible by the possibility of on the fly evaluation of alternative scenarios and solution frameworks based on different levels and kinds of process, and product, innovation.

A costly trial and error process can be shortened working on virtual models of the different mechatronic system alternative layouts.

In summary the key objectives pursued by means of the simulator tool are:

- Validation of the garment assembly line 'holistic concept': first of all the viability of the proposed technologies and solutions must be checked roughly on the basis of their guessed characteristics (timings, capacities etc...)

- Layout definition against realistic production data: after that the core choices have been done, the design specification are checked quantitatively against realistic production scenarios drawn from current state of the art manufacturing practices

- Progressive development of the model adopting what-if approach: the possible design choices are evaluated in advance quantitatively against the desired performance indexes

- Prediction of realistic production metrics after line deployment: assumed and defined the design parameters the future production scenarios can be checked

- Validation of 'operation procedures' (how production is managed): in the varied operation condition during the life of the manufacturing facility many scenario can occur: from a scheduled maintenance to a subsystem failure. 'Operation procedures' have to be defined in order to properly manage any foreseeable occurrence. A realistic simulation model allows to test and compare in advance the operation practices.

- Ergonomics and labor effort analyses: the tools allow to verify some labor efforts and to know in advance excessive requests on the workers.

- Visualization of the model as a virtual reality environment: it also provides a quick 'at glance' preview of how an envisioned solution could work

It is also devised that the GALsim simulator will be used as a decision support tool in order to improve the utilization of the line $[5,7,10,11]$.

This also needs paying a special attention to the easiness of maintenance in order to be able to evaluate in a reasonable time the different production scheduling alternatives. 


\section{GARMENT ASSEMBLY LINE}

As told above the garment assembly line is conceived as a complex mechatronic system conceived in order to cope with the flexibility needs of the emerging mass customization scenario. In the figure below (fig. 1) we can show the layout of the garment assembly line as it is represented by the GALsim system.

The 'holistic' MES (Manufacturing Execution System) is conceived as a multiagent intelligent modular system.

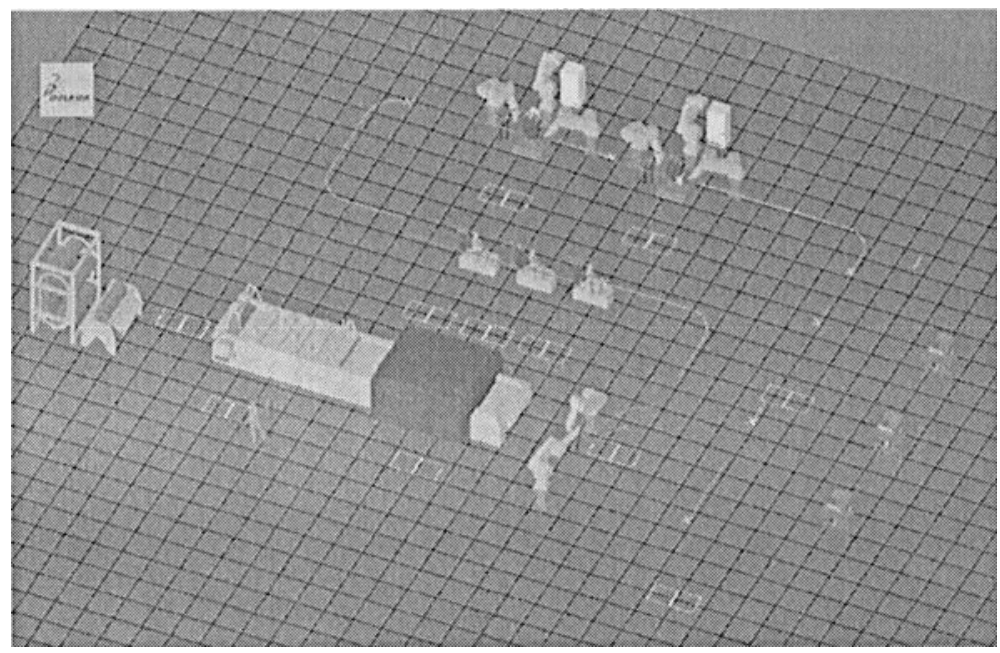

Figure 1 - Garment Assembly Line Layout

The entities constituting the line are:

- Cutting table: a digital single-ply multiple head cutting table which cut the fabric rolls in order to obtain the 'cut parts', the pieces of fabrics which are sewed together

- Fusing

- Unloading of Cutting Table: it is performed by robots through particular advanced grippers

- 2D Automatic Sewing Machines they are dedicated to the sewing of some specific subassemblies like sleeves

- Garment Assembly Cell (GAC): this cell is constituted by a reconfigurable mould, by dedicated positioning equipment

- 2D Manual Sewing: these are quite classical sewing machines like those currently used for most sewing operation of the cloth manufacturing cycle.

In Figure 2 is summarized the multiagent structure of the MES system. 


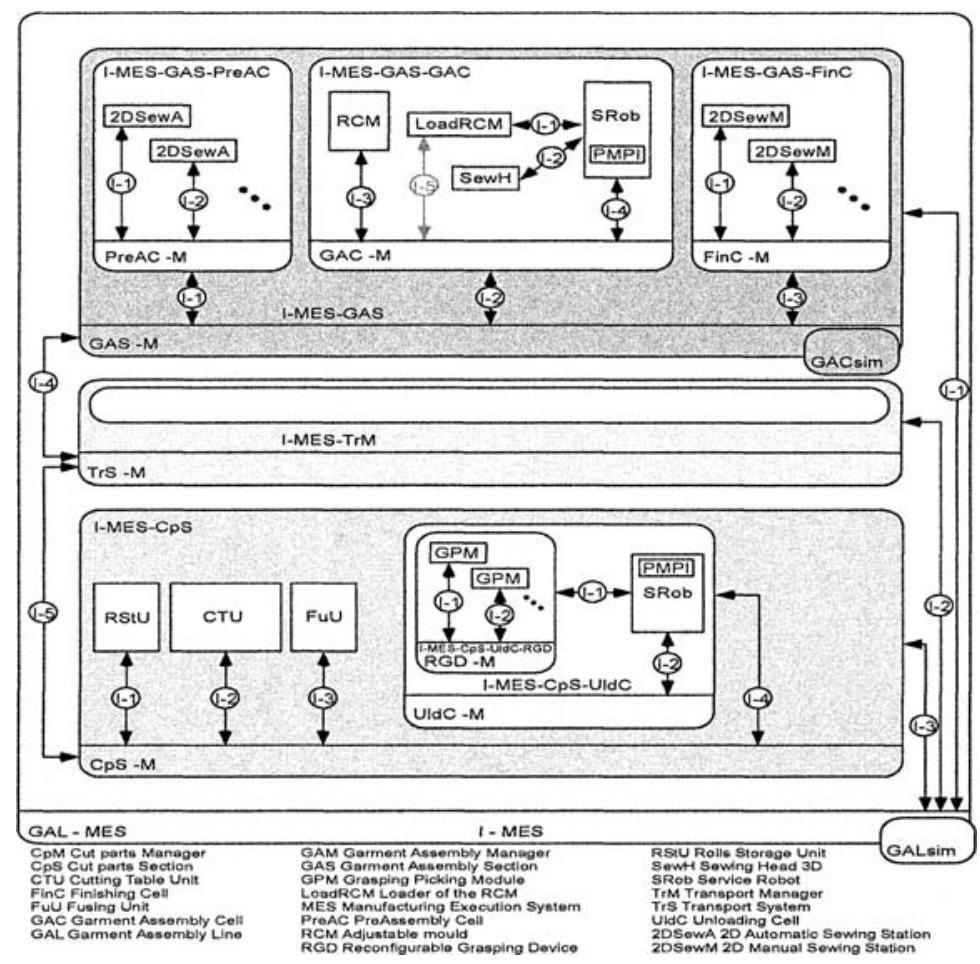

Figure 2 - MES Multiagent structure

\section{SIMULATION MODEL}

The modeling of the system requires that we properly define the part model, the resource model and the process model.

The part model is the modeling of raw materials, semi-finished, finished goods that flow through the plant.

The resource model is the modeling of machines, transport systems, buffers.

The process model is the modeling of the manufacturing operations defining how operations are performed in the machines, how transportations are managed in the transport systems, how buffers are managed, in general all the rules governing the evolution of the garment assembly line.

\subsection{Part Model}

The part model requires that are defined the items ('parts') flowing through the line, as number and kind.

The reference finished good item considered is the formal men jacket.

The main kinds of items we have considered are: Fabric rolls, Cut parts, Formal jacket sub assemblies, and Formal jackets. 


\subsection{Resource Model}

The resource model requires that we define the resources by which the items are transformed and moved from a place to another in the plant.

The kinds of resources include various types of machines (cutting table, loading/unloading robots, sewing robots, reconfigurable moulds, 2D sewing automated machines), human operators, transportation systems.

For any resource a number of parameters must be defined, regarding: set up/operation times; logics, capacity, etc.; input type (push/pull); output type ( push/pull ); cycle process (times of operation/operation-steps, ...); setup process: set up (lot/n of pieces), timings; unloading process; part routing (depending on layout topology); MTTR, MTBF; Shifts

The statuses that can be assumed by the resources are: Idle, Busy, Blocked, Failed, Off shifts.

\subsection{Process Model}

The modeling of the manufacturing facility requires that the process model is defined, i.e. the definition of logic rules stating the reciprocal interaction of elements in the model.

The flow of production is simulated with an event driven approach, defined by means of resource attributes.

It is necessary to define:

- Dispatching rules: e.g., first available machine, minimum set up time, priority

- Connections between the resources

- Priorities

The dispatching rules and connections are between involved resources.

\subsection{Simulation Outputs}

As we have seen in section 1.1, GALsim, as a core design tool for a complex system that cannot be represented in closed form, allows to compare the effects of different design choices and to assess the alternative technologies (for example for the reconfigurable mould).

The reporting capabilities allow to identify: bottlenecks, responsiveness to failures, throughput, resource saturation, labor effort, queue lengths/times, work in process.

It is possible to show VR animation of garment assembly process evolution during the simulated time, updating the reports and diagrams online.

In the figures below some examples of output: two VR animation snapshot pictures, in fig 3 (left), the line before the start of the shift of the workers at the manual sewing cells, in fig 3 (right) the line during a cut table failure; in fig. 4 (up), an example of utilization pie chart of the cut table around a failure, in fig. 4 (down) a snapshot of a body jacket subassemblies present in the Garment Assembly Cell at a given time. 
It must be noticed that the snapshots in fig. 3 are VR animation snapshots, the view point can be moved quite freely allowing to see the scenes from different perspectives, this is particularly useful when analysing ergonomics (for the workers) or mobility issues (for the robots).
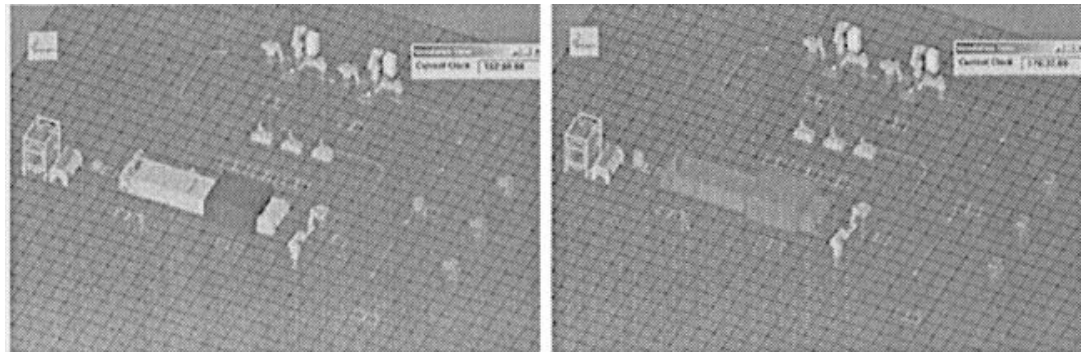

Figure 3 - Line snapshot before worker shift begin (left), line snapshot in failure condition (right)

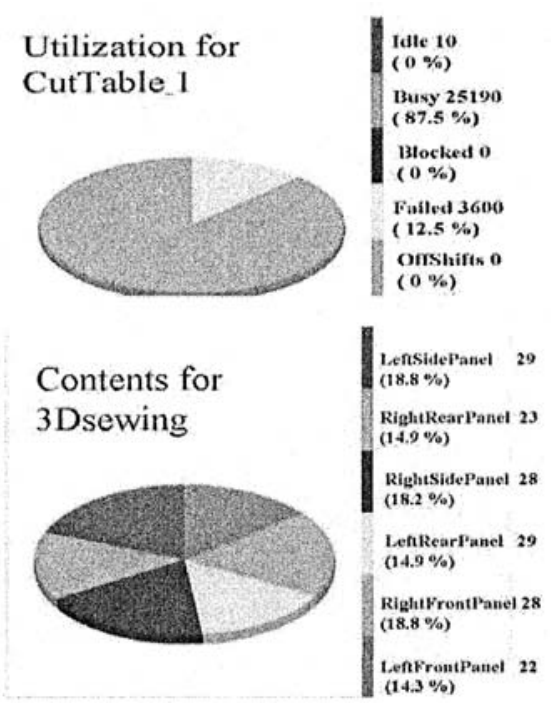

Figure 4 - Utilization for Cut Table (up), 3Dsewing part content snapshot (down)

\section{FURTHER DEVELOPMENTS AND CONCLUSIONS}

Current version of GALsim allows to simulate production management of the overall system in the different conditions envisioned at design time and to provide an operational decision support system after the facility deployment. 
A detailed mechanical simulator of the most critical activities of handling and grasping from the cutting table, of loading unloading on the transport system and of the body jacket $3 \mathrm{D}$ sewing will be developed. It will be integrated seamlessly with the general simulator of garment assembly line.

A new concept textile manufacturing facility design substantially reshapes the current cloth manufacturing methodologies.

The resulting multiagent holistic mechatronic production facility represents an example of complex non linear system.

A new paradigm garment assembly line design requires new resource and production management models, thus requiring a new dedicated simulation tool.

An advanced Virtual Reality simulation tool provides the necessary quantitative support for the analysis of design alternatives and for the assessment of proposed technologies.

\section{ACKNOWLEDGMENTS}

The Leapfrog (Leadership for European Apparel Production From Research along Original Guidelines) project is funded by the EC under the FP6-2003-NMP-NI-3, Proposal no 515810-2.

\section{REFERENCES}

1. Aguiar, M.W.C., Murgatroyed, I.S., Edwards, J.E. 1996. "Object-oriented resource models: their role in specfying components in integrated manufacturing systems", Computer Aided Manufacturing Systems, Vol $9, \mathrm{~N}^{\circ} 1$.

2. Chalmeta, R., Williams, T.J., Lario, F. and Ros, L. 1997. "Developing an Object-Oriented Reference Model for Manufacturing", in Manufacturing Systems: modelling, management and control, P. Kopacek, Ed., Preprints of the IFAC Workshop on Manufacturing Systems: modelling, management and control (Vienna, Austria, Feb. 3-5). 379-384

3. Leondes, T. (Ed.) Computer Aided and Integrated Manufacturing Systems Techniques and Applications, Vol. II: Computer-Integrated Manufacturing (Boca Raton, Florida: CRC Press LLC, 2001).

4. Moss, S., Davidsson, P. Multi-Agent-Based Simulation, Springer, Mar 1, 2001

Appa Iyer Sivakumar, Chin Soon Chong, Development of an Object Oriented Simulation Engine for On-Line Simulation and Optimization, 33rd Annual Simulation Symposium pp. 291-299

5. McNally, P., Heavey, C. Developing simulation as a desktop resource. International Journal of Computer Integrated Manufacturing, Volume 17, Number 5 / July-August 2004, pp. 435 - 450

6. Acaccia, Chiavacci, Michelini, Callegari, Benchmarking clothing industry: effectiveness by computer simulation," Proc. of 11 th European Symposium: Simulation in Industry ESS-99, Erlangen, October 26-28, 1999, pp. 519-524.

7. Acaccia, Conte, Maina, Michelini, "Computer aids for intelligent manufacture of quality clothes," Computer in Industry 50 (1) 2003, pp. 71-84.

8. Acaccia, Conte, Maina, Michelini, Molfino, "Integrated manifacture of high standing dresses for customized satisfaction," Globalisation of Manifacturing in the digital communication eta, KLUWER, Boston, 1999, pp.511-523.

9. Bruzzone, L., Molfino, R.M., Zoppi, M. A discrete event simulation package for modular and adaptive assembly plants. In M.H. Hamza, editor, 22nd Int. Conf. Modelling, Identification and Control MIC2003, pages 280-282, Innsbruck, Austria, February 10-13 2003. IASTED/ACTA Press.

10. Bonsignorio F., Ferrari R., Busatti M., Bottero M. "A simulation tool integrated in a short term planning system", in PROCEEDINGS OF THE 4TH INTL. CONF. ON SIMULATION IN MANUFACTURING, London 1988, IFS

11. Bonsignorio F., Ferrari R., Trebino O., "Practical integration of a short term planning system in a production management information system", in Proceedings of the 1989 Intl. Workshop on Decisional Structures in Automated Manufacturing, Genova 1989, IFAC-CIRP-IFIP- IFORS 